\title{
Development and testing quantitative metrics from multi-parametric magnetic resonance imaging that predict Gleason score for prostate tumors
}

\author{
Rulon Mayer ${ }^{1,2}$, Charles B. Simone $\mathrm{II}^{3}$, Baris Turkbey ${ }^{4}$, Peter Choyke ${ }^{4}$ \\ ${ }^{1}$ University of Pennsylvania, Philadelphia, PA, USA; ${ }^{2}$ OncoScore, Garrett Park, MD, USA; ${ }^{3}$ New York Proton Center, New York, NY, USA; \\ ${ }^{4}$ National Institutes of Health, Bethesda, MD, USA
}

Contributions: (I) Conception and design: R Mayer; (II) Administrative support: R Mayer, CB Simone 2nd, P Choyke; (III) Provision of study materials or patients: B Turkbey, P Choyke; (IV) Collection and assembly of data: R Mayer; (V) Data analysis and interpretation: R Mayer; (VI) Manuscript writing: All authors; (VII) Final approval of manuscript: All authors.

Correspondence to: Rulon Mayer, PhD. University of Pennsylvania, Philadelphia, PA 19104, USA; OncoScore, Garrett Park, MD 20896, USA.

Email: mayerru@yahoo.com; mayerru@gmail.com.

Background: Radiologists currently subjectively examine multi-parametric magnetic resonance imaging (MRI) to detect possible clinically significant lesions using the Prostate Imaging Reporting and Data System (PI-RADS) protocol. The assessment of imaging, however, relies on the experience and judgement of radiologists creating opportunity for inter-reader variability. Quantitative metrics, such as z-score and signal to clutter ratio (SCR), are therefore needed.

Methods: Multi-parametric MRI (T1, T2, diffusion, dynamic contrast-enhanced images) were resampled, rescaled, translated, and stitched to form spatially registered multi-parametric cubes for patients undergoing radical prostatectomy. Multi-parametric signatures that characterize prostate tumors were inserted into z-score and SCR. The multispectral covariance matrix was computed for the outlined normal prostate. The z-score from each MRI image was computed and summed. To reduce noise in the covariance matrix, following matrix decomposition, the noisy eigenvectors were removed. Also, regularization and modified regularization was applied to the covariance matrix by minimizing the discrimination score. The filtered and regularized covariance matrices were inserted into the SCR calculation. The z-score and SCR were quantitatively compared to Gleason scores from clinical pathology assessment of the histology of sectioned wholemount prostates.

Results: Twenty-six consecutive patients were enrolled in this retrospective study. Median patient age was 60 years (range, 49 to 75 years), median prostate-specific antigen (PSA) was $5.8 \mathrm{ng} / \mathrm{mL}$ (range, 2.3 to $23.7 \mathrm{ng} / \mathrm{mL}$ ), and median Gleason score was 7 (range, 6 to 9). A linear fit of the summed z-score against Gleason score found a correlation of $\mathrm{R}=0.48$ and a $\mathrm{P}$ value of 0.015 . A linear fit of the SCR from regularizing covariance matrix against Gleason score found a correlation of $\mathrm{R}=0.39$ and a $\mathrm{P}$ value of 0.058 . The SCR employing the modified regularizing covariance matrix against Gleason score found a correlation of $\mathrm{R}=0.52$ and a $\mathrm{P}$ value of 0.007 . A linear fit of the SCR from filtering out 3 and 4 eigenvectors from the covariance matrix against Gleason score found correlations of $\mathrm{R}=0.50$ and 0.44 , respectively, and $\mathrm{P}$ values of 0.011 and 0.027 , respectively.

Conclusions: Z-score and SCR using filtered and regularized covariance matrices derived from spatially registered multi-parametric MRI correlates with Gleason score with highly significant P values.

Keywords: Supervised target detection; adaptive cosine estimator; prostate cancer (PCa); multi-parametric magnetic resonance imaging (multi-parametric MRI); Gleason score; histology of wholemount prostatectomy 
Submitted Aug 11, 2021. Accepted for publication Oct 18, 2021.

doi: 10.21037/qims-21-761

View this article at: https://dx.doi.org/10.21037/qims-21-761

\section{Introduction}

Clinical management of prostate cancer (PCa) critically depends on knowing the cancer's potential aggressiveness and ability to metastasize (1-3). PCa staging and nomograms that predict clinical outcomes, such as biochemical recurrence after treatment (4-6), conventionally use a wide variety of clinical indicators (4-17), including prostatespecific antigen (PSA) (7-9), seminal vesicle involvement $(10,11)$, tumor volume (12-16), magnetic resonance imaging (MRI) (17-21), cancer spread $(10,11,14)$, and Gleason score (22) after biopsy or prostatectomy. Some of this input information, like PSA (8), is not consistently predictive of outcome. Other input, such as assessment of imaging or histology slices, rely on the experience and judgement of radiologists and pathologists (17-20), creating opportunity for inter-reader variability. A quantitative approach could reduce such variability.

PCa has been studied using multi-parametric MRI (MPMRI) via the Prostate Imaging Reporting and Data System (PI-RADS) protocol (17-20). In the PI-RADS approach, radiologists apply a series of learned rules to the visualized $\mathrm{PCa}$ to generate a PI-RADS score for the lesion. Currently research (20) employing the PI-RADS approach successfully assesses tumors for lymph node involvement, extra-prostatic extension, and clinically significant disease, but only weakly correlates with Gleason score. A more quantitative and less subjective approach for assessing MP-MRI may raise the evaluation accuracy and consistency.

Recently (23-25), pilot studies of hyperspectral target detection techniques were applied to hypercubes derived from spatially registering MP-MRI. These studies predicted Gleason score using image-based signatures, measured tumor volume, and calculated tumor eccentricity or shape and correlated with Gleason score. Instead of comparing image-based signatures (23) to determine Gleason score, this study uses the spatially registered hypercubes to compute the degree of disease through the summed z-score and signal to clutter ratio (SCR) and relates them to Gleason score. For each modality in MP-MRI, z-score and SCR computes the ratio of the tumor signal value minus the mean background (or normal prostate) to the standard deviation of normal prostate. Normalizing the tumor signal partially mitigates (23) varying MRI pulse sequences, magnetic fields, injection of contrast, etc. among patients. Normalizing to this background presumes that the variation within a given patient's normal prostates are roughly similar for all patients. Z-score and SCR differ in how they gather the information from among the different MP-MRI modalities.

The goal of this study to see if quantitative measures from spatially registered MP-MRI such as z-score and modified versions of SCR are correlated with Gleason score, a proven indicator of clinical outcome. The goal is to develop a non-invasive, objective measure for assessing $\mathrm{PCa}$ and provide guidance for disease management. This is merely a retrospective pilot study that will hopefully lead to more definitive research.

We present the following article in accordance with the TRIPOD reporting checklist (available at https://dx.doi. org/10.21037/qims-21-761).

\section{Methods}

\section{Overall method description}

Figure 1 shows the overall scheme to compare Gleason score derived from histology with z-score and SCR generated from spatially registered MRI. Gleason score is determined from pathology analysis of the histopathology slides from radical prostatectomy (blue arrows). In the MP-MRI arm of the study (red arrows), spatially registered hypercubes are assembled from the individual MRI pulse sequences. The prostate is outlined and in scene signatures are derived from the hypercube and provide input for the z-score and SCR computation. Noise in the SCR is reduced. The z-score and SCR following noise reduction through eigenvalue filtering and also regularizing the covariance matrix $(\mathrm{CM})$ is compared to the histology-based Gleason score.

\section{Study design and population}

The Cancer Imaging Archive (TCIA) $(26,27)$ affiliated with The National Institutes of Health (NIH) stored collected patient data from prostate tumor MRI and histology from wholemount prostatectomy specimens. The local institutional review board (IRB) approved this publicly 


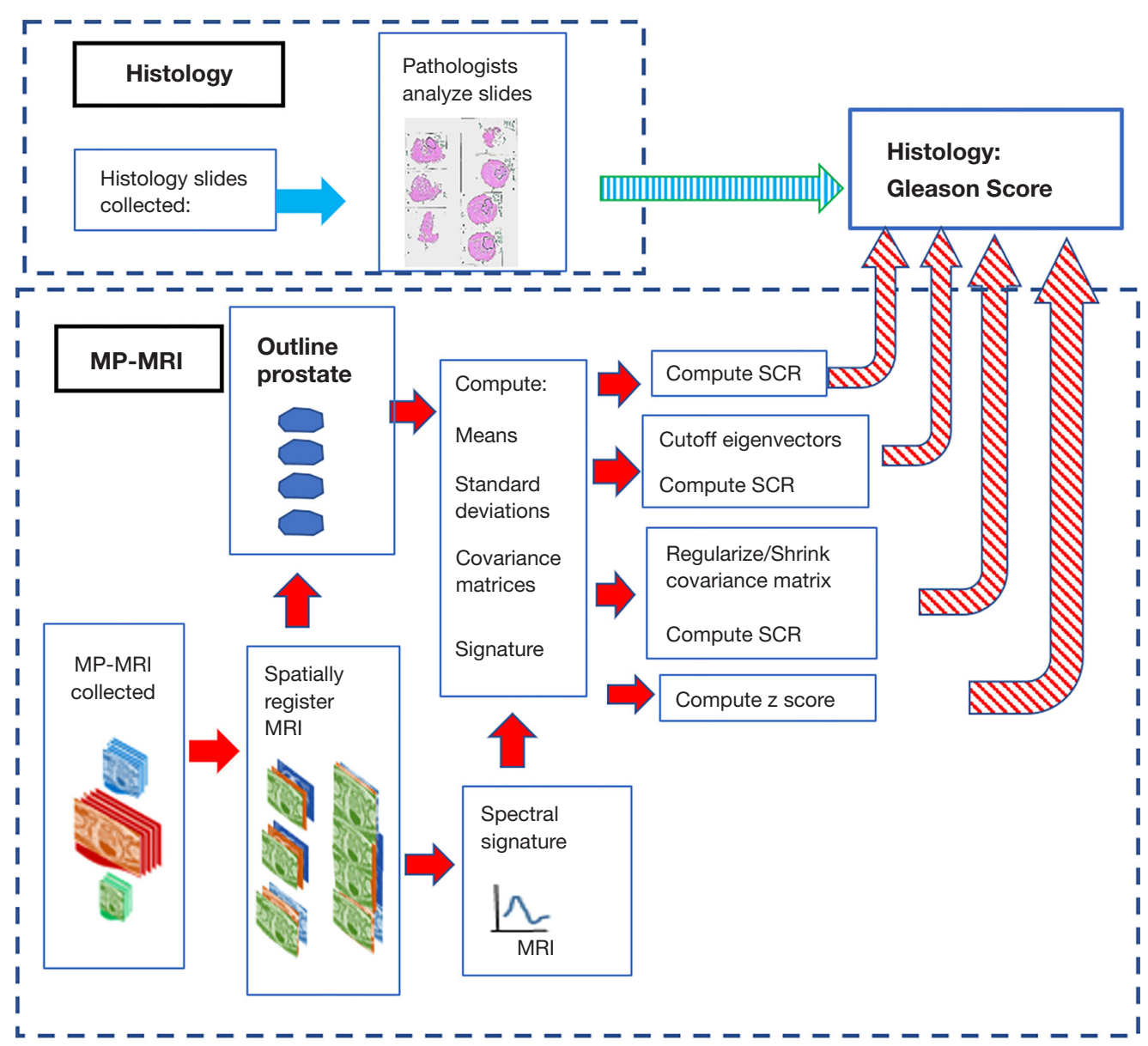

Figure 1 Overall scheme to compare Gleason score derived from histology (blue arrows) with z-score and SCR generated from spatially registered MRI (red arrows). SCR, signal to clutter ratio; MP-MRI, multi-parametric magnetic resonance imaging.

available, single institution retrospective study. The study was conducted in accordance with the Declaration of Helsinki (as revised in 2013). Due to previous protections implemented by TCIA, this retrospective, anonymized study was exempt from further IRB review and individual consent for this retrospective analysis was waived.

\section{Whole mount prostatectomy and histology}

Three-dimensional (3D) models of each prostate $(28,29)$ were generated by segmenting the prostate capsule on in vivo triplane T2-weighted (T2W) MRI, and extracting from the high-resolution 3D surfaces. Commercially available 3D computer aided design software helped design each mold. The design accounted for the deformation of the endorectal coil. A 3D printer (Dimension Elite 3D printer, Stratasys, Inc.) deposited styrene to fabricate each mold. The specimen was fixed at room temperature in formalin for 2 to $24 \mathrm{~h}$ after radical prostatectomy, then placed in the customized 3D mold, and sliced in axial $6 \mathrm{~mm}$ sections. Using the customized mold for histopathology, the whole mount histopathology patient specimens were sectioned corresponding to the axial plane of the MRI sections. Two experienced pathologists independently, and blinded from MRI analysis, shortly after the prostatectomy in 2008 to 2009, mapped the slides for individual tumor foci, dimensions, and classified the tumor blobs into Gleason scores, not the International Society of Urological Pathology (ISUP) grading. Individual blobs in a given patient's histology slide can have differing Gleason scores. As in previous efforts (23-25) and to better reflect the patient's status, a patient's Gleason score is a weighted 
average (based on histology blob size) of the Gleason scores assessed by the pathologist. Future studies with greater patient samples may find that ISUP correlates better with $\mathrm{z}$-scores and SCR.

\section{MRI}

The MRI collection is composed of Diffusion Weighted Images (DWI), dynamic contrast enhanced (DCE), and structural (T1, T2) images. The pulse sequences were described in earlier studies (27-29). These studies (28-30) were performed with an endorectal coil (BPX-30, Medrad; Pittsburgh, PA, USA) tuned to $127.8 \mathrm{MHz}$ and a 16-channel cardiac coil (SENSE, Philips Medical Systems; Best, the Netherlands). The MRI used a 3 Tesla (T) magnet (Achieva, Philips Medical Systems). Prior bowel preparation was not used. Standard methods were used to insert the endorectal coil. The MRI protocol used triplanar T2W turbo spin echo, DW MRI, 3DMR point resolved spectroscopy, and axial pre-contrast $\mathrm{T} 1$-weighted axial 3D fast field echo DCE MRI sequences. Their detailed sequence parameters were defined in a prior study (29).

\section{Image processing, pre-analysis}

The presence of elevated vasculature that feeds the rapidly growing tumor helps distinguish lesions from normal tissues. DCE displays the time evolution of contrast material over several hundred seconds after its injection and shows uptake in the tissues. The vasculature is porous to contrast material. The contrast material enters the tumor's extravascular space (but not the cells) and can fill and empty of MRI contrast material more quickly than the normal prostate organ. DCE analysis and exploitation of the unique tumor physiology can identify a portion of the tumors. A simple two compartment model $(31,32)$ describes the tracer concentration in the tissue that supplies and empties through the tumor vasculature. The model is used to create the washout $\mathrm{k}_{\mathrm{ep}}$ image.

In the superior-inferior direction, the slices were resampled to 6-mm spacing based on the patient's table position. Fine-tuned rigid registration (minor transverse translation, resampling) was applied between the structural, diffusion, and DCE due to the short time intervals between scans (<20 minutes). The MRI images were digitally resampled (23-25) to $1-\mathrm{mm}$ resolution in the transverse direction. Individual slices were scaled, translated, resliced and spatially registered at the pixel level to create a "cube".
Multiple axial cubes in three dimensions were "stitched" together by sequentially connecting them together into a narrow 3D image. Four dimensions (3D body volume plus the fourth dimension composed of MRI sequences) object is thereby re-expressed as a $3 \mathrm{D}$ mosaicked cube. Each voxel is treated as a vector composed of MRI modality rather than a scalar value. Multispectral MRI data contain 7 components (23-25) [T1 (pre contrast), T1 (maximum contrast), T2, automated diffusion coefficient (ADC), DWI-high B $\left(\mathrm{B}=1,000 \mathrm{~s} / \mathrm{mm}^{2}\right)$, Washout or $\mathrm{k}_{\mathrm{ep}}$ from DCE].

\section{Overall quantitative metrics description: $z$-score and SCR}

To generate a quantitative metric (33-36) for assessing the degree of disease (and used in many fields), a z-score is computed using the digital values from MRI. Figure 2 schematically illustrates the z-score computation for three modalities, specifically Washout or exit rate of contrast material derived from kinematic analysis of DCE (Figure $2 A, 2 D$ ), the "high B" component of the DWI (Figure $2 B, 2 E$ ), and ADC (Figure 2C,2E). These three MRI modalities have been spatially registered to each other. The red cross hairs in Figure $2 A-2 C$, the horizontal profiles in Figure $2 D-2 F$, and the downward pointing arrows in Figure $2 A-2 E$ correspond to the same set of point(s) in the patient's body (horizontal pixel 120 in Figure $2 D-2 F$ ). Prostate tumors show hyperintense regions in the Washout (Figure 2A) and DWI (Figure 2B) and hypointense region in the ADC (Figure 1C). The red crosshairs in Figure $2 A$ $2 C$ denote a point in the prostate tumor. Prostate tumor exhibits an elevated value $S_{i}-\mu_{i}$ (solid green) relative the prostate mean MRI $\mu_{\mathrm{i}}$ (solid blue line) and the added standard deviation $\sigma_{\mathrm{i}}$ (dashed green line) (see Eq. [2]).

Specifically, the z_score for given for each modality $i$ is (36):

$$
z_{-} \text {score }_{i}=\left(\frac{S_{i}-\mu_{i}}{\sigma_{i}}\right)^{2}
$$

and $i$ ranges from $i=1,2,3 \ldots M\left(M=7\right.$ in this study), where $S_{i}$ is the tumor signature or value in the modality $\mathrm{i}$ (mean over the $T$ tumor pixels), $\mu_{\mathrm{i}}$ is the mean value for normal prostate in MRI modality i, where

$$
S_{i}=\frac{1}{T} \sum_{q}^{T} x_{i, q}, \mu_{i}=\frac{1}{N} \sum_{p}^{N} x_{i, p}, \sigma_{i}=\frac{1}{N}\left(\sum_{p}^{N}\left(x_{i, p}-\mu_{i}\right)^{2}\right)^{1 / 2}[2]
$$

and $\sigma_{\mathrm{i}}$ is the standard deviation for the normal prostate in modality $\mathrm{i}$, and $\mathrm{p}$ ranges over all $\mathrm{N}$ prostate voxels. $\mathrm{S}$, the in-scene tumor signature, is selected from yellow voxels in a 
A

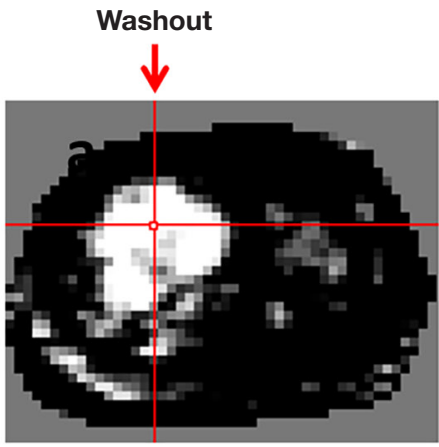

D

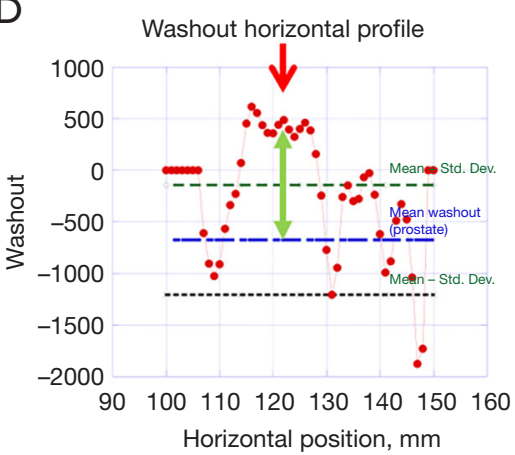

B

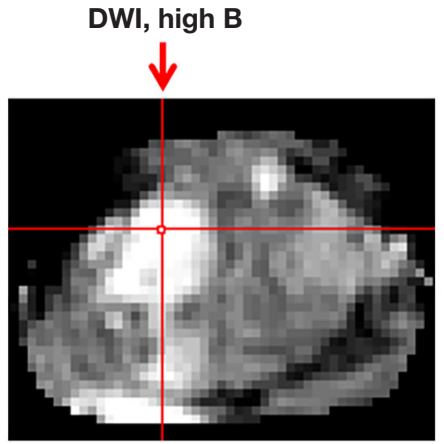

$\mathrm{E}$

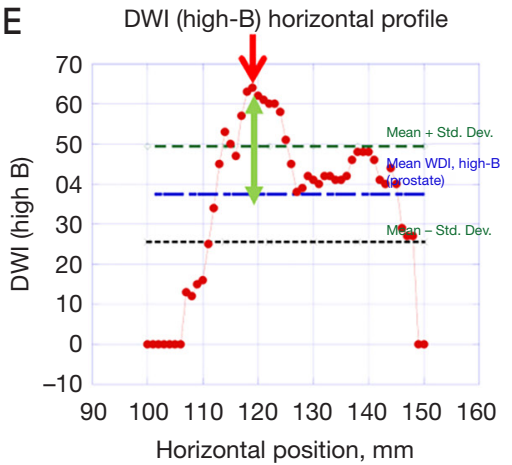

C

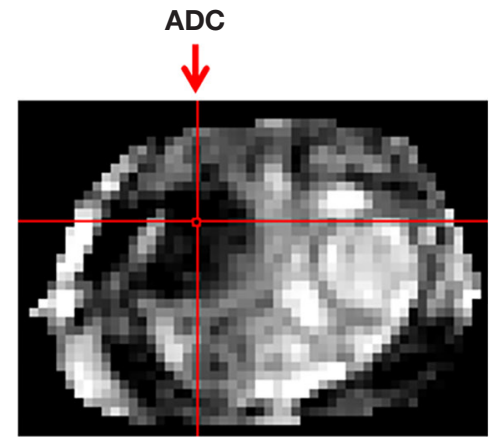

$\mathrm{F}$

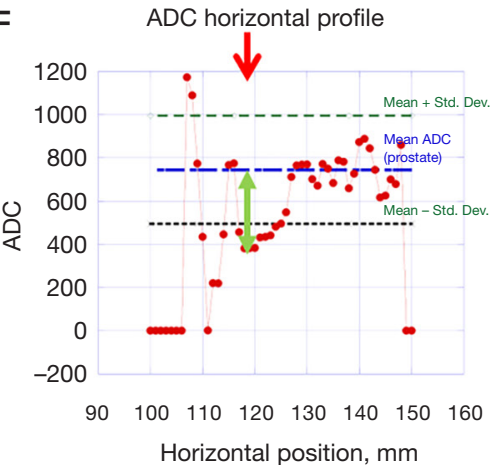

Figure 2 The z-score computation for three modalities, specifically Washout or exit rate of contrast material derived from kinematic analysis of dynamic contrast enhancement images (DCI) (A,D), the "high B" component of the diffusion weighted image (DWI) (B,E), and automated diffusion coefficient (ADC) (C,F).

three-color display of the spatially-registered MP-MRI (red is Washout, green is DWI, high-B, blue is ADC) (23-25). The background voxels needed for $\mu$ and $\mathrm{CM}$ were taken from digitally outlining the prostate on the spatiallyregistered MP-MRI. A simple way of combining all MPMRI modalities $i$ is to sum the individual $z$-score ${ }_{i}$, i.e.,

$$
z_{-} \text {score }=\sum_{i}^{M} z_{-} \text {score }_{i}
$$

The $z$ score (Eq. [3]) is the Euclidean distance (3436) between the tumor or target and the prostate or background. The simple sum of $z$-score e $_{i}$ (Eq. [2]) from each modality $i$ is only one quantitative metric that incorporates all MP-MRI contributions into characterizing the prostate tumor. Another, the SCR (32-34) is given by

$$
S C R=(S-\mu)^{T} C M^{-1}(S-\mu)
$$

that is a matrix multiplication over MP-MRI modalities, the superscript $\mathrm{T}$ denotes a vector transpose operation, $\mathrm{CM}$ is the covariance matrix, and the superscript -1 denotes a matrix inverse operation. Note: the SCR (Eq. [4]) is commonly expressed in decibels $(\mathrm{dB})[\mathrm{SCR}(\mathrm{dB})=20$ $\log 10(\mathrm{SCR})]$. The $\mathrm{CM}_{\mathrm{i}, \mathrm{j}}$ is given by

$$
C M_{i, j}=\sum_{p}^{N}\left(x_{p, i}-\mu_{i}\right)\left(x_{p, j}-\mu_{j}\right)
$$

for the sum over $\mathrm{N}_{\mathrm{i}}$ and $\mathrm{x}_{\mathrm{j}}$ voxels in the prostate for MRI modalities i and j. The SCR (Eq. [4]) is the Mahalanobis distance (34-36) or Euclidean distance in the "whitened space" between tumor and normal prostate.

$\mathrm{CM}$ is a symmetric square $\mathrm{M} \times \mathrm{M}$ matrix and, therefore, diagonalizable (37) and composed of variations (cross variations) along the diagonal (non-diagonal) directions. SCR (Eq. [4]) is identical to z-score (Eq. [3]) if CM is the identity matrix.

After diagonalization, eigenvector generation, the whitening transform linearly combines, and rotates the M MRI modalities. The SCR (Eq. [4]) maximizes the $\mathrm{z}$-score. However, the CM contains noise components resulting in inaccurate and undesired quantitative metrics (38). To reduce the CM noise, two options are invoked, namely filtering out the high noise/low value eigenvalue components (section "Filtering noise") $(38,39)$, and regularizing or shrinking the CM (section 


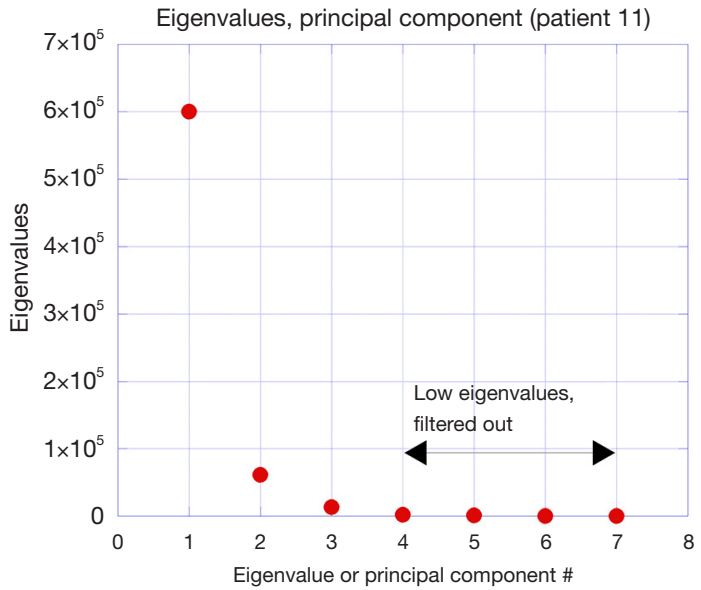

Figure 3 Eigenvalues from analyzing the covariance matrix for a spatially registered 7 components MP-MRI from patient \#11. Lowest value eigenvalues are filtered out of covariance matrix to remove noise. MP-MRI, multi-parametric magnetic resonance imaging.

"Regularization and shrinkage") (38).

\section{Filtering noise}

To reduce the noise, image information is decorrelated by using principal component analysis and removing the noise in the low eigenvalue channels $(38,39)$. CM is a square symmetrical matrix that is decomposed into three parts (37),

$$
C M=\Lambda^{T} \lambda \Lambda
$$

Similarly, the inverse covariance matrix $\mathrm{CM}^{-1}$ is a square symmetrical matrix that is decomposed into three parts,

$$
C M^{-1}=\Lambda^{T} \lambda^{-1} \Lambda
$$

namely the eigenmatrix $\Lambda$, transpose of the eigenmatrix $\Lambda^{\mathrm{T}}$, and diagonal matrix $\lambda$ with eigenvalues $\lambda^{2}{ }_{1}, \lambda^{2}{ }_{2}, \lambda^{2}{ }_{3} \ldots \lambda^{2}{ }_{M}$ populating the diagonal

$$
\lambda^{-1}=\left[\begin{array}{ccccc}
\frac{1}{\lambda_{1}^{2}} & 0 & \ldots . . & 0 & 0 \\
0 & \frac{1}{\lambda_{2}^{2}} & 0 & & 0 \\
\ldots & & \ldots & & \ldots \\
0 & & & \frac{1}{\lambda_{M-1}^{2}} & 0 \\
0 & & & 0 & \frac{1}{\lambda_{M}^{2}}
\end{array}\right]
$$

Conventionally, the eigenvalues are ordered according to size ranging from the largest $\lambda_{1}$ to the smallest $\lambda_{\mathrm{M}}$. The images corresponding to the eigenvalues and eigenvectors range from high signal and variation $(1,2)$ to low variation and very noisy $(M-1, M)$. The eigenvalues span a huge range of values. The lowest value eigenvalues $(M-1, M)$ elevate the noise due to the inversion in the inverse matrix $\mathrm{CM}^{-1}$ (Eq. [8]). Figure 3 depicts a specific display of eigenvalues from analyzing the $\mathrm{CM}$ for a spatially registered 7 component MP-MRI from a particular patient and masking for prostate. Filtering out the noisy eigenvectors means removing the lowest valued eigenvalues from the inverse matrix, i.e.,

$$
\lambda_{\text {Filtered }}^{-1}=\left[\begin{array}{ccccc}
\frac{1}{\lambda_{1}^{2}} & 0 & . . & 0 & 0 \\
0 & \frac{1}{\lambda_{2}^{2}} & 0 & & 0 \\
\ldots & & \ldots & & \ldots \\
0 & & & 0 & 0 \\
0 & 0 & \ldots . & 0 & 0
\end{array}\right]
$$

and inserting Eq. [9] into Eq. [7]

$$
C M_{\text {Filtered }}^{-1}=\Lambda^{T} \lambda_{\text {Filtered }}^{-1} \Lambda
$$

resulting in

$$
S C R_{\text {Filtered }}=(S-\mu)^{T} C M_{\text {Filtered }}^{-1}(S-\mu)
$$

After inserting Eq. [10] into Eq. [4].

\section{Regularization and shrinkage}

Another approach for reducing noise is through regularization and shrinkage (38). That is shrinking the difference in the highest and lowest eigenvalues by adding a diagonal component that is controlled by the parameter $\gamma$ and thereby perturbing the $\mathrm{CM}$ into the regularized $\mathrm{CM}_{\text {Reg }}(\gamma)$

$$
C M_{\mathrm{Reg}}(\gamma)=(1-\gamma) C M+\frac{\gamma \operatorname{Tr} a(C M)}{M} I
$$

by control value $\gamma$ where Tra denotes the trace operator and $\mathrm{I}$ is the identity matrix. $\gamma$ ranges from $\gamma=0.0$ or no $\mathrm{CM}$ modification to $\gamma=1.0$ or $\mathrm{CM}$ is proportional to the identity matrix. The goal of regularization is to perturb the $\operatorname{CM}(\gamma)$ so as to maximize the normal distribution, or equivalently minimize the discriminant function $\mathrm{d}(\gamma)[=-\ln$ (normal distribution)], i.e., 


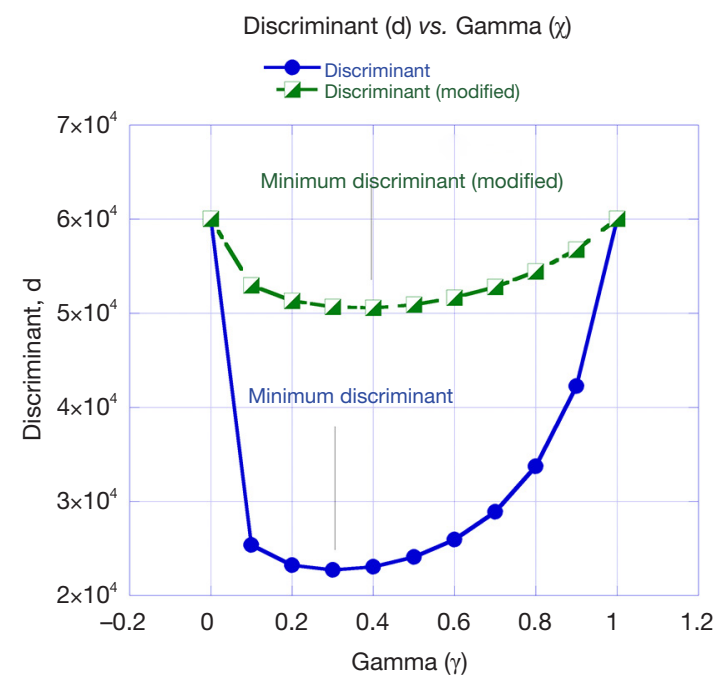

Figure 4 Discriminant and modified discriminant function for patient \#11 as a function of $\gamma$. Minimum discriminant $(\gamma=0.30)$ and modified discriminant $(\gamma=0.40)$ denoted by red and blue arrows, respectively.

$$
\begin{aligned}
d_{\mathrm{Reg}}(\gamma)= & \sum_{i}^{N}\left(x_{i}-\mu\right)^{T} C M_{\mathrm{Reg}}(\gamma)^{-1}\left(x_{i}-\mu\right) \\
& +\ln \left(\operatorname{det}\left(C M_{\mathrm{Reg}}(\gamma)\right)\right)
\end{aligned}
$$

where the sum is over all $\mathrm{N}$ samples in the prostate ensemble and det denotes the determinant operation. A search is conducted among the $\gamma$ 's (range $0<\gamma<1$ ) for the lowest discriminant function that can be achieved with $\gamma_{\min }$ and results in a regularized $\mathrm{SCR}_{\mathrm{Reg}}$ :

$$
S C R_{\mathrm{Reg}}\left(\gamma=\gamma_{\text {Min }}\right)=(S-\mu)^{T} C M_{\mathrm{Reg}}^{-1}\left(\gamma=\gamma_{\text {Min }}\right)(S-\mu)
$$

A less well studied $\mathrm{CM}$ regularization $\mathrm{CM}_{\text {mod }}(\gamma)$ instead corrects the CM by

$$
C M_{\text {mod_Reg }}(\gamma)=(1-\gamma) C M+\gamma V
$$

$\mathrm{V}$ is not the identity matrix I but instead is a diagonal matrix filled up with the square of the standard deviations from $M$ modalities and is given by

$$
V=\left[\begin{array}{ccccc}
\sigma_{1}^{2} & 0 & \ldots & 0 & 0 \\
0 & \sigma_{2}^{2} & & & 0 \\
\ldots . & & \ldots & & \ldots \\
0 & & & \sigma_{M-1}^{2} & 0 \\
0 & 0 & \ldots & 0 & \sigma_{M}^{2}
\end{array}\right]
$$

And using Eqs. $[15,16]$ the modified discriminant function $\mathrm{d}_{\text {mod_Reg }}(\gamma)$ is

$$
\begin{aligned}
d_{\text {mod_Reg }}(\gamma) & =\sum_{i}^{N}\left(x_{i}-\mu\right)^{T} C M_{\text {mod_Reg }}(\gamma)\left(x_{i}-\mu\right) \\
& +\ln \left(\operatorname{det}\left(C M_{\text {mod_Reg }}(\gamma)\right)\right)
\end{aligned}
$$

is computed for $0<\gamma<1$. A minimum $d_{\text {mod }}\left(\gamma_{\min }\right)$ is found at $\gamma_{\min }$. resulting in a $\mathrm{SCR}_{\text {mod_Reg }}$ (Eq. [18]) using a modified regularization procedure (using Eqs. [15-17]).

$$
S C R_{\text {mod_Reg }}\left(\gamma=\gamma_{\text {min }}\right)=(S-\mu)^{T} C M_{\text {mod_Reg }}^{-1}\left(\gamma=\gamma_{\text {min }}\right)(S-\mu)
$$

It should be noted that $\gamma=0$ results in the standard SCR (Eq. [4]) and $\gamma=1$ results in the z-score (Eq. [3]).

Figure 4 shows the dependence of discriminant function $\mathrm{d}_{\text {Reg }}(\gamma)$ (Eq. [13]) and $\mathrm{d}_{\text {mod_Reg }}(\gamma)($ Eq. [17]) on the parameter $\gamma$ for patient \#11. The minimum discriminant function and modified discriminant function in this case occurs at $\gamma=0.30$ and $\gamma=0.40$, respectively.

\section{Results}

A total of 26 consecutive patients enrolled in the study between July 2008 and July 2009. All patients showed biopsy-proven adenocarcinoma of the prostate. Median patient age was 60 years (range, 49 to 75 years). Median PSA was $5.8 \mathrm{ng} / \mathrm{mL}$ (range, 2.3 to $23.7 \mathrm{ng} / \mathrm{mL}$ ). Median Gleason score was 7 (range, 6 to 9). No restrictions were placed on tumor location within the prostate. A robotic assisted radical prostatectomy was performed at a median time of 60 days (range, 3 to 180 years) after the MRI without any intervening treatment. The histology assessment of the prostate specimen was done right after the prostatectomy. One patient was excluded due to failure to uptake the MRI contrast material. Table 1 summarizes relevant patient features for this study including Gleason score, contrast uptake, and tumor volume derived from analysis of the histology of wholemount prostatectomy.

From analysis of 25 patients (1 patient excluded due to no contrast uptake), Figure 5 shows the Pearson correlation coefficient $\mathrm{R}$ (red, filled dot) and $\mathrm{P}$ values (blue, halffilled square) for fitting filtered SCR (Eq. [11]) against the Gleason score. The correlation (shown in red) shows a maximum value, whereas the $\mathrm{P}$ value shows a minimum. Cutting off 3 and 4 of the lowest value eigenvectors from the $\mathrm{CM}$ calculation yielded acceptable $(\mathrm{P}<0.05)$ filtered $\mathrm{SCR}_{\text {Filter }}$ for fitting to the Gleason score. The Pearson 
Table 1 Summary of patient characteristics (Gleason score, contrast uptake, volume)

\begin{tabular}{|c|c|c|c|}
\hline Patient \# & Gleason score & Contrast uptake & Histology volume (cc) \\
\hline 1 & 6.67 & Yes & 0.745 \\
\hline 2 & 8.67 & Yes & 18.168 \\
\hline 3 & 6.8 & Yes & 2.970 \\
\hline 4 & 7.73 & Yes & 12.620 \\
\hline 5 & 7.87 & Yes & 5.310 \\
\hline 6 & 7.47 & Yes & 2.350 \\
\hline 7 & 8.44 & Yes & 3.160 \\
\hline 8 & 6.75 & Yes & 1.040 \\
\hline 9 & 6.39 & Yes & 4.057 \\
\hline 10 & 6.27 & Yes & 0.697 \\
\hline 11 & 7.83 & Yes & 3.215 \\
\hline 12 & 6.50 & Yes & 2.325 \\
\hline 13 & 8.00 & Yes & 1.270 \\
\hline 14 & 6.4 & Yes & 5.134 \\
\hline 15 & 6.67 & Yes & 1.600 \\
\hline 16 & 6.67 & Yes & 1.150 \\
\hline 17 & 6.67 & Yes & 1.900 \\
\hline 18 & 6.67 & Yes & 5.470 \\
\hline 19 & 6.67 & Yes & 2.540 \\
\hline 20 & 7.33 & Yes & 4.947 \\
\hline 21 & 6.67 & Yes & 1.210 \\
\hline 22 & 6.67 & Yes & 2.270 \\
\hline 23 & 6.67 & Yes & 0.420 \\
\hline 24 & 8.33 & No & 1.260 \\
\hline 25 & 6.89 & Yes & 1.049 \\
\hline 26 & 6.89 & Yes & 0.570 \\
\hline
\end{tabular}

correlation coefficients for 3 and 4 remaining eigenvectors were 0.44 and 0.50 , respectively. The $\mathrm{P}$ values for 3 and 4 eigenvectors were 0.027 and 0.015 , respectively. Table 2 displays a summary for Pearson correlation coefficient and $\mathrm{P}$ values of cutoffs with Gleason score.

Figure 6 shows a scatterplot (25 patients) of filtered

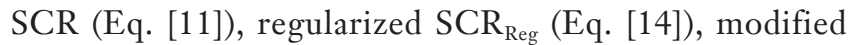
regularized $\mathrm{SCR}_{\text {mod_Reg }}$ (Eq. [18]), and z-score (Eq. [3]) against Gleason score. Processing 25 patients, yielded the Pearson correlation coefficients from fitting the histology

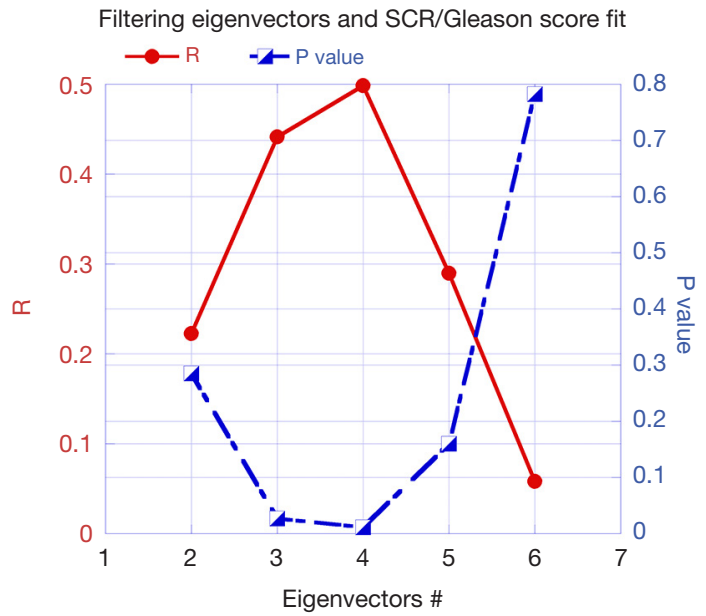

Figure 5 Correlation coefficient (R) (red, filled dot) and $\mathrm{P}$ values (blue, half-filled square) for fitting filtered SCR and Gleason score. SCR, signal to clutter ratio.

Table 2 Summary of Pearson correlation coefficient (R) and P values for MP-MRI and Gleason score

\begin{tabular}{lcc}
\hline Variables & $\begin{array}{c}\text { Correlation } \\
\text { coefficient (R) }\end{array}$ & P value \\
\hline Cutoff (3 remaining eigenvectors) & 0.44 & 0.027 \\
Cutoff (4 remaining eigenvectors) & 0.50 & 0.011 \\
Regularization & 0.38 & 0.058 \\
Modified regularization & 0.52 & 0.007 \\
Z-score & 0.48 & 0.015 \\
\hline
\end{tabular}

R, Pearson correlation coefficient; MP-MRI, multi-parametric magnetic resonance imaging.

derived Gleason scores to MP-MRI-based z-score, $\mathrm{SCR}_{\mathrm{Reg}}$ from regularized covariance, $\mathrm{SCR}_{\text {mod_Reg }}$ from modified regularized $\mathrm{CM}$, and $\mathrm{SCR}_{\text {Filter }}$ from filtering out 4 eigenvectors $0.48,0.52,0.39$, and 0.50 , respectively. Regularization and shrinkage and modified regularization calculation for 25 patients yielded an average minimum $\gamma$ 's of $\gamma_{\min }=0.316 \pm 0.0464$ and $\gamma_{\min }=0.373 \pm 0.0452$, respectively.

In addition, the $\mathrm{P}$ values from comparing histology derived Gleason scores and MP-MRI-based z-score, $\mathrm{SCR}_{\mathrm{Reg}}$ from regularized covariance, modified regularized $\mathrm{SCR}_{\text {mod_Reg }}$ and $\mathrm{SCR}_{\text {Filter }}$ from filtering out 4 eigenvectors are 0.015 , $0.052,0.007$, and 0.011 , respectively. Table 2 summarizes the Pearson correlation coefficient and $\mathrm{P}$ values of $\mathrm{z}$-score and regularization with Gleason score. 


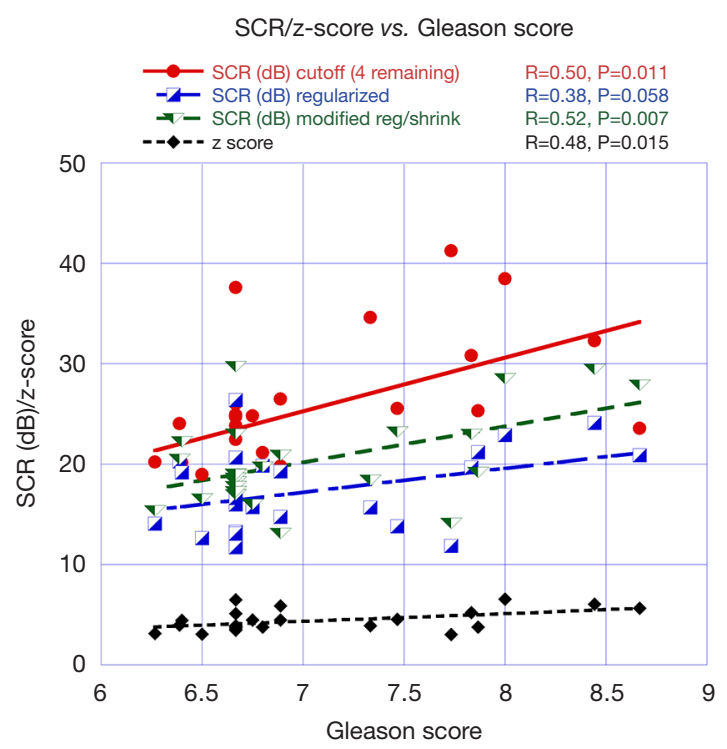

Figure 6 Scatter plot for 25 patients SCR with cutoff filter (red, filled dot), SCR regularized (blue, half-filled square), SCR modified regularized (green, half triangle), and Z-score (black, filled diamond) against Gleason scores. Correlation coefficients $\mathrm{R}$ and $\mathrm{P}$ values also shown. SCR, signal to clutter ratio.

\section{Discussion}

The metrics, z-score and SCR that use the regularized, modified regularized, and filtered CM, correlate with the Gleason score. For the SCR calculation, however, it is essential to reduce the noise by filtering or regularizing the CM. Inserting unregularized CM into the SCR calculation yields huge ratios albeit with poor correlation with Gleason score. By deriving quantitative metrics from digital values from the images instead of evaluating the visual appearance of the image, it is hoped that reliable assessments of patient images can accurately ascertain a patient's disease status.

Although the Pearson correlation coefficients between z-score, SCR and Gleason score are not close to 1.0, they remain statistically significant with low $\mathrm{P}$ values. To provide perspective, depending on the study, the far better studied connection between PSA and Gleason score Pearson correlation coefficient varies from $\mathrm{R}=0.59$ (8) to $\mathrm{R}=0.43-0.46$ (9) to having $\mathrm{P}=0.75$ and showing no statistical significance (7). The connection between PIRADS and Gleason score also shows variation among studies. Bastian-Jordan (18) demonstrated the incidence of clinically significant disease for different PI-RADS grades. PI-RADS score was not associated with significant differences regarding Gleason score distribution within the target (19). However, the relationships between PIRADS score and postoperative extracapsular extension, lymphovascular invasion, and seminal vesicle involvement were significant $(\mathrm{P}<0.001, \mathrm{P}=0.032$, and $\mathrm{P}=0.007$, respectively) (20).

A previous pilot study used image-based transformed spectral signatures (24) derived from spatially registered MP-MRI to predict Gleason scores. This current study instead uses z-scores and modified SCR to determine Gleason scores. Analysis of spatially-registered MP-MRI offers two independent direct measures (Gleason score signatures and z-score/SCR) to assess Gleason scores. These measures may complement and supplement more indirect indicators of tumor aggressiveness such as tumor volume and eccentricity. It may be clinically advantageous to combine predictions from signatures and z-scores/modified SCR in a complementary fashion such as in a nomogram or a multivariable fit.

No algorithm proscribes the cutoff for filtering eigenvalues. An empirical approach $(38,39)$, as in this study, determines the optimal filtered value by comparing the SCR calculation to Gleason score. The cutoff is often chosen to reside at the "shoulder" in the eigenvalues to filter out the noise. The eigenvalue number cutoff of 3 or 4 found in this study resides at the shoulder (see Figure 3 as an example), following previous efforts.

Regularization of the CM is often invoked in order to mitigate the rank deficiency matrix singularity due to undersampling relative to the number of features or components (38). In this study, however, the number of samples (roughly 2,000) far exceeds the 7 components. Nevertheless, regularization and modified regularization greatly increased the correlation between Gleason score and SCR.

The modified regularized CM is not well studied relative to the regularized CM (38). This modified regularized CM was previously suggested for study but not examined (38). Figure 4 shows the discriminant function for the modified regularized covariance is larger than the discriminant function for the ordinary regularized covariance. However, employing the modified regularization relative to the customary regularization resulted in increasing the Pearson correlation coefficient ( $\mathrm{R}=0.38$ to 0.52$)$ and reducing the $\mathrm{P}$ value ( $\mathrm{P}=0.058$ to 0.007$)$ in this study. To understand the result, it should be noted that $\gamma=1$ for the ordinary regularization procedure (Eqs. $[12,13])$ results in 


$$
S C R_{\mathrm{Reg}}(\gamma=1)=\sum_{i}^{M}\left(\frac{\left(S_{i}-\mu_{i}\right)}{\sigma_{\text {Mean }}}\right)^{2}
$$

where $\sigma_{\text {mean }}$ corresponds to the mean standard deviation $\left(\operatorname{Tra}(\mathrm{CM}) / \mathrm{M}\right.$, Eq. [12]). Eq. [19] uses a single value of $\sigma_{\text {mean }}$ rather than $M$ different $\sigma_{\mathrm{i}}$ 's. Evidently, it is important to correct for variations within each MRI modality through the individual standard deviations (Eqs. $[1,3,18]$ with $\gamma=1.0$ ) in order to predict the Gleason score. This study combines different modalities with significantly varying standard deviations. The standard deviation for images from different wavelengths in hyperspectral imagery, however, do not vary much and so normal regularization may be sufficient, unlike this study.

To optimize regularization and modified regularization, this study varied the $\gamma$ to search for the minimum discriminant score (Eqs. $[13,17])$. The search can be computationally burdensome. Due to the narrow range of the minimum $\gamma$ 's for regularization and modified regularization $\left(\gamma_{\min }=0.316 \pm 0.0464\right.$ and $\left.\gamma_{\min }=0.373 \pm 0.0452\right)$ and the broad minimum in the discriminant functions, it may be advantageous to choose a single $\gamma, \gamma=0.3$ and $\gamma=0.4$ for regularization and modified regularization, respectively. Choosing a single $\gamma$ instead of searching for an optimal $\gamma$ should speed up the calculation resulting in minimal loss of accuracy and precision.

If additional studies (using greater number of patients, conducted prospectively) confirm that SCR/z-scores, eccentricity significantly correlate with Gleason score, then clinical implementation is feasible. The biggest issue is generating spatially registered hypercubes from MP-MRI in a timely manner. However, software development should enable autonomous spatial registration that would reduce the time for assembling the hypercubes. Identifying tumors by the radiologist to generate tumor signature is simplified for spatial-registered hypercubes by using color processing previously discussed in an earlier study (25). The clinical implementation is non-invasive and reduces possible side effects for the patient.

The eigenvalue filtering and covariance regularization processes discussed in this paper are not the only way to reduce noise or singularities in the CM (40). The other approaches more directly reduce [relative to regularization (38)] the difference in the disparity between the highest and lowest eigenvalues. Such other approaches may generate improvement in correlating CM-based approaches with Gleason score and should be investigated.

This study suffers from a number of limitations.
The patients from this study originated from a single institution (NIH). Variations in clinical implementation were, therefore, not examined and its effect on this study is uncertain. This is a retrospective, not a prospective study thereby limiting the conclusions. Although a small number of patients were assessed, consecutive patients were analyzed and statistically significant $\mathrm{P}$ values showing potential clinical value were achieved.

\section{Conclusions}

Z-score and SCR using filtered and regularized covariance matrices derived from spatially registered MP-MRI correlates with Gleason score with statistically significant low $\mathrm{P}$ values.

\section{Acknowledgments}

Funding: None.

\section{Footnote}

Reporting Checklist: The authors have completed the TRIPOD reporting checklist. Available at https://dx.doi. org/10.21037/qims-21-761

Conflicts of Interest: All authors have completed the ICMJE uniform disclosure form (available at https://dx.doi. org/10.21037/qims-21-761). The authors have no conflicts of interest to declare.

Ethical Statement: The authors are accountable for all aspects of the work in ensuring that questions related to the accuracy or integrity of any part of the work are appropriately investigated and resolved. The study was conducted in accordance with the Declaration of Helsinki (as revised in 2013). This retrospectively designed, single institution study was approved by the NIH Institutional Review Board, and was compliant with the Health Insurance Portability and Accountability Act. And individual consent for this retrospective analysis was waived.

Open Access Statement: This is an Open Access article distributed in accordance with the Creative Commons Attribution-NonCommercial-NoDerivs 4.0 International License (CC BY-NC-ND 4.0), which permits the noncommercial replication and distribution of the article with the strict proviso that no changes or edits are made and the 
original work is properly cited (including links to both the formal publication through the relevant DOI and the license). See: https://creativecommons.org/licenses/by-nc-nd/4.0/.

\section{References}

1. Sobin L, Wittekind C. TNM classification of malignant tumors. 5th edition. New York: John Wiley and Sons, Inc., 1997.

2. Shariat SF, Karakiewicz PI, Roehrborn CG, Kattan MW. An updated catalog of prostate cancer predictive tools. Cancer 2008;113:3075-99.

3. Martin NE, Mucci LA, Loda M, Depinho RA. Prognostic determinants in prostate cancer. Cancer J 2011;17:429-37.

4. Ho R, Siddiqui MM, George AK, Frye T, Kilchevsky A, Fascelli M, Shakir NA, Chelluri R, Abboud SF, Walton-Diaz A, Sankineni S, Merino MJ, Turkbey B, Choyke PL, Wood BJ, Pinto PA. Preoperative Multiparametric Magnetic Resonance Imaging Predicts Biochemical Recurrence in Prostate Cancer after Radical Prostatectomy. PLoS One 2016;11:e0157313.

5. May M, Siegsmund M, Hammermann F, Loy V, Gunia S. Visual estimation of the tumor volume in prostate cancer: a useful means for predicting biochemical-free survival after radical prostatectomy? Prostate Cancer Prostatic Dis 2007;10:66-71.

6. Poulakis V, Witzsch U, de Vries R, Emmerlich V, Meves M, Altmannsberger HM, Becht E. Preoperative neural network using combined magnetic resonance imaging variables, prostate-specific antigen, and gleason score for predicting prostate cancer biochemical recurrence after radical prostatectomy. Urology 2004;64:1165-70.

7. Gurumurthy D, Maggad R, Patel S. Prostate carcinoma: correlation of histopathology with serum prostate specific antigen. Science Journal of Clinical Medicine 2015;4:1-5

8. Ngwu PE, Achor GO, Eziefule VU, Orji JI, Alozie FT. Correlation between Prostate Specific Antigen and Prostate Biopsy Gleason Score. Annals of Health Research 2019;5:243-8.

9. Zivkovic S. Correlation between prostate-specific antigen and histopathological difference of prostate carcinoma. Arch Oncol 2004;12:148-51.

10. Wang L, Hricak H, Kattan MW, Chen HN, Kuroiwa K, Eisenberg HF, Scardino PT. Prediction of seminal vesicle invasion in prostate cancer: incremental value of adding endorectal MR imaging to the Kattan nomogram. Radiology 2007;242:182-8.

11. Villers AA, McNeal JE, Redwine EA, Freiha FS, Stamey
TA. Pathogenesis and biological significance of seminal vesicle invasion in prostatic adenocarcinoma. J Urol 1990;143:1183-7.

12. McNeal JE. Cancer volume and site of origin of adenocarcinoma in the prostate: relationship to local and distant spread. Hum Pathol 1992;23:258-66.

13. Friedersdorff F, Groß B, Maxeiner A, Jung K, Miller K, Stephan C, Busch J, Kilic E. Does the Prostate Health Index Depend on Tumor Volume?-A Study on 196 Patients after Radical Prostatectomy. Int J Mol Sci 2017;18:488.

14. McNeal JE, Villers AA, Redwine EA, Freiha FS, Stamey TA. Histologic differentiation, cancer volume, and pelvic lymph node metastasis in adenocarcinoma of the prostate. Cancer 1990;66:1225-33.

15. Epstein JI, Carmichael M, Partin AW, Walsh PC. Is tumor volume an independent predictor of progression following radical prostatectomy? A multivariate analysis of 185 clinical stage B adenocarcinomas of the prostate with 5 years of followup. J Urol 1993;149:1478-81.

16. Kikuchi E, Scardino PT, Wheeler TM, Slawin KM, Ohori $M$. Is tumor volume an independent prognostic factor in clinically localized prostate cancer? J Urol 2004;172:508-11.

17. Weinreb JC, Barentsz JO, Choyke PL, Cornud F, Haider MA, Macura KJ, Margolis D, Schnall MD, Shtern F, Tempany CM, Thoeny HC, Verma S. PI-RADS Prostate Imaging-Reporting and Data System:2015, Version 2. Eur Urol 2016;69:16-40.

18. Bastian-Jordan M. Magnetic resonance imaging of the prostate and targeted biopsy, Comparison of PIRADS and Gleason grading. J Med Imaging Radiat Oncol 2018;62:183-7.

19. Slaoui $H$, Neuzillet $Y$, Ghoneim $T$, Rouanne $M$, Abdou A, Lugagne-Delpon PM, Scherrer A, Radulescu C, Delancourt C, Molinié V, Lebret T. Gleason Score within Prostate Abnormal Areas Defined by Multiparametric Magnetic Resonance Imaging Did Not Vary According to the PIRADS Score. Urol Int 2017;99:156-61.

20. Kızılay F, Çelik S, Sözen S, Özveren B, Eskiçorapçı S, Özgen M, Özen H, Akdo an B, Aslan G, Narter F, Çal Ç, Türkeri L; Members of Urooncology Association. Correlation of Prostate-Imaging Reporting and Data Scoring System scoring on multiparametric prostate magnetic resonance imaging with histopathological factors in radical prostatectomy material in Turkish prostate cancer patients: a multicenter study of the Urooncology Association. Prostate Int 2020;8:10-5. 
21. Shukla-Dave A, Hricak H, Kattan MW, Pucar D, Kuroiwa K, Chen HN, Spector J, Koutcher JA, Zakian KL, Scardino PT. The utility of magnetic resonance imaging and spectroscopy for predicting insignificant prostate cancer: an initial analysis. BJU Int 2007;99:786-93.

22. Gleason DF, Mellinger GT. Prediction of prognosis for prostatic adenocarcinoma by combined histological grading and clinical staging. J Urol 1974;111:58-64.

23. Mayer R, Simone CB 2nd, Turkbey B, Choyke P. Correlation of prostate tumor eccentricity and Gleason scoring from prostatectomy and multi-parametricmagnetic resonance imaging. Quant Imaging Med Surg 2021;11:4235-44.

24. Mayer R, Simone CB 2nd, Skinner W, Turkbey B, Choykey P. Pilot study for supervised target detection applied to spatially registered multiparametric MRI in order to non-invasively score prostate cancer. Comput Biol Med 2018;94:65-73.

25. Mayer R, Simone CB 2nd, Turkbey B, Choyke P. Algorithms applied to spatially registered multi-parametric MRI for prostate tumor volume measurement. Quant Imaging Med Surg 2021;11:119-32.

26. Choyke P, Turkbey B, Pinto P, Merino M, Wood B. Data From PROSTATE-MRI. The Cancer Imaging Archive, 2016. Available online: http://doi.org/10.7937/K9/ TCIA.2016.6046GUDv

27. Clark K, Vendt B, Smith K, Freymann J, Kirby J, Koppel P, Moore S, Phillips S, Maffitt D, Pringle M, Tarbox L, Prior F. The Cancer Imaging Archive (TCIA): maintaining and operating a public information repository. J Digit Imaging 2013;26:1045-57.

28. Shah V, Pohida T, Turkbey B, Mani H, Merino M, Pinto PA, Choyke P, Bernardo M. A method for correlating in vivo prostate magnetic resonance imaging and histopathology using individualized magnetic resonancebased molds. Rev Sci Instrum 2009;80:104301.

29. Turkbey B, Mani H, Shah V, Rastinehad AR, Bernardo M, Pohida T, Pang Y, Daar D, Benjamin C, McKinney YL, Trivedi H, Chua C, Bratslavsky G, Shih JH, Linehan WM, Merino MJ, Choyke PL, Pinto PA. Multiparametric
$3 \mathrm{~T}$ prostate magnetic resonance imaging to detect cancer: histopathological correlation using prostatectomy specimens processed in customized magnetic resonance imaging based molds. J Urol 2011;186:1818-24.

30. Turkbey B, Pinto PA, Mani H, Bernardo M, Pang Y, McKinney YL, Khurana K, Ravizzini GC, Albert PS, Merino MJ, Choyke PL. Prostate cancer: value of multiparametric MR imaging at $3 \mathrm{~T}$ for detection-histopathologic correlation. Radiology 2010;255:89-99.

31. Tofts PS, Brix G, Buckley DL, Evelhoch JL, Henderson E, Knopp MV, Larsson HB, Lee TY, Mayr NA, Parker GJ, Port RE, Taylor J, Weisskoff RM. Estimating kinetic parameters from dynamic contrast-enhanced T(1)-weighted MRI of a diffusable tracer: standardized quantities and symbols. J Magn Reson Imaging 1999;10:223-32.

32. Tofts PS. T1-weighted DCE Imaging Concepts: Modelling, Acquisition and Analysis. Magnetom Flash 2010;3:31-9.

33. Jain AK. Fundamentals of Digital Image Processing. Upper Saddle River, NJ: Prentice Hall, 1989.

34. Richards JA, Jia X. Remote Sensing Digital Image Analysis. 3rd edition. New York: Springer-Verlag, 1999.

35. Manolakis D, Shaw G. Detection algorithms for hyperspectral imaging applications. IEEE Signal Processing Magazine 2002;19:29-43.

36. Duda RO, Hart PE, Stork DG. Pattern Classification. (2nd edition. New York: Wiley, 2001.

37. Strang G. Linear algebra and its applications. 4th edition. Belmont, CA: Thomson, Brooks/Cole, 2006.

38. Friedman JH. Regularized Discriminant Analysis. J Am Stat Assoc 1989;84:165-75.

39. Chen G, Qian S. Denoising of Hyperspectral Imagery Using Principal Component Analysis and Wavelet Shrinkage. IEEE Transactions on Geoscience and Remote Sensing 2011;49:973-80.

40. James W, Stein C. Estimation with Quadratic Loss. Proceedings of the Fourth Berkeley Symposium Mathematical Statistics and Probability. Berkeley: University of California Press, 1961;1:361-79.
Cite this article as: Mayer R, Simone CB 2nd, Turkbey B, Choyke P. Development and testing quantitative metrics from multi-parametric magnetic resonance imaging that predict Gleason score for prostate tumors. Quant Imaging Med Surg 2022;12(3):1859-1870. doi: 10.21037/qims-21-761 